\title{
Consequências e Características de um Processo de Desenvolvimento de Software de Qualidade e Aspectos que o influenciam: uma avaliação de especialistas
}

\author{
Jeann M. Andrade, Adriano B. Albuquerque, Fábio B. Campos, Ana Regina C. Rocha \\ \{jeann, bessa, Bianchi, arocha\}@cos.ufrj.br \\ Programa de Engenharia de Sistemas de Computação - COPPE/UFRJ
}

\begin{abstract}
Resumo
A sistematização do desenvolvimento pelo uso de processos de engenharia de software tem o potencial de trazer vários benefícios para as organizações desenvolvedoras de software, porém, caracterizar objetivamente o que é um processo efetivo e eficaz não é tarefa simples. Esta caracterização se mostra importante em vários momentos: na definição do processo adequado à organização e seus projetos; na implantação desse processo; no acompanhamento dos seus resultados e na sua melhoria. Visando iniciar essa caracterização, foi realizada uma pesquisa (survey) onde foram apresentados aos participantes, três grupos distintos de questões relativas a processos de software, que buscam responder às seguintes perguntas: Que benefícios o uso de processos de engenharia de software pode trazer? Quais são as principais características que um processo efetivo deve ter? e, por último, Que aspectos organizacionais, humanos e técnicos podem influenciar para a efetividade dos processos?. Os resultados consolidados da pesquisa são apresentados, permitindo uma análise preliminar de quais seriam aqueles aspectos mais relevantes a se considerar na definição, uso e melhoria de processos.
\end{abstract}

Palavras-chaves: Processo de Software, Melhoria de Processos, Tomada de Decisões, Qualidade de Software.

\begin{abstract}
The systematization of software development through the use of software engineering processes has the power to bring many benefits to software development organizations, however, characterizing objectively, what is an effective and efficient process is not a simple task. This characterization is important in many times: in the definition of an adequate process to the organization and its projects; in the deployment of this process; in the monitoring of its results and in its improvement. To initiate this characterization, a survey was conducted, presenting three distinct groups of questions related to software processes, that aimed to answer the following questions: What benefits the use of a software engineering process can bring? Which are the main characteristics that an effective process must have?, and, finally, Which organizational, human and technician aspects can influence the effectiveness of the processes. The consolidated results of the research are presented, allowing a preliminary analysis of which aspects would be more relevant to be considered in the definition, utilization and improvement of processes.
\end{abstract}

Key-Words: Software Process, Process Improvement, Decision making, Software Quality.

\section{Introdução}

Produtos de software estão presentes na sociedade das mais diversas formas. Segundo REED [1] se alguns sistemas de uso global deixarem de funcionar, aproximadamente $40 \%$ da população mundial sofrerá as conseqüências do problema.

No Brasil, observa-se também o crescimento da indústria de software e a importância que a mesma está representando para a economia. De acordo com a publicação "Qualidade e Produtividade no Setor de Software Brasileiro" [2], o valor total da comercialização bruta de software das organizações da amostra apurado para o ano 2000 foi de R 1.684 milhões, onde $70 \%$ resultou de software por encomenda (R\$ 1.185 milhões). Os domínios de software que mais se destacaram na pesquisa, dentre vinte categorias definidas, foram: administração 
privada (42\%), serviços em geral (38\%), setor financeiro e indústria (35\%), comércio $(34 \%)$ e administração pública (33\%).

As organizações de software que não conseguirem: maior qualidade nos seus produtos, tornando-os confiáveis, maior produtividade, redução de custos e melhoria na previsibilidade dos seus projetos, correm grandes riscos de não mais permanecerem no mercado.

Diante deste contexto, a indústria de software e a academia estão, cada vez mais, investindo em processos de software, apostando neste recurso como um meio para alcançar algumas das exigências do mercado relacionadas acima. Além disso, há grandes evidências de que a qualidade de um produto de software está fortemente relacionada com a qualidade dos processos que foram utilizados para desenvolvê-lo. No entanto, para que um processo de software seja realmente efetivo para os seus projetos, é importante considerar vários aspectos.

O objetivo do presente trabalho é identificar o que a comunidade de engenharia de software considera como conseqüências de um bom processo para projetos de software, as características de um bom processo de software e os aspectos que podem influenciar positivamente e negativamente na utilização do processo.

O resultado deste trabalho auxiliará as organizações a conseguirem obter um maior sucesso na implantação e utilização de seus processos de desenvolvimento de software, levando-as, principalmente, a conhecerem os riscos que devem ser prioritariamente administrados, bem como os pontos de melhoria mais relevantes.

Este trabalho está organizado da seguinte forma: a seção 2 apresenta, resumidamente, conceitos de processo de software, melhoria de processos e seus benefícios; a seção 3 mostra a metodologia utilizada na pesquisa; a seção 4 apresenta os resultados obtidos, que são analisados na seção 5 e a seção 6 apresenta as conclusões deste trabalho e suas perspectivas de continuidade.

\section{Processo de Software e Melhoria do Processo}

As organizações têm dado uma maior ênfase na utilização de processos de software como uma das formas de buscar, principalmente, uma maior qualidade e controle sobre custos e prazos dos projetos.

Processo de Software pode ser definido como: um conjunto de atividades, métodos, práticas e transformações que pessoas utilizam para desenvolver e manter software e seus produtos associados [3].

Para ZAHRAN [4], a melhoria da qualidade, seja em processos de desenvolvimento de software, seja em qualquer outra área, está intimamente ligada à necessidade de adaptação da empresa com ênfase em produto, para a empresa focada em processos. $\mathrm{O}$ autor aponta os seguintes benefícios, quando o foco dos projetos está no processo: alinhamento entre as atividades e o foco comum; consistência entre as várias atividades; possibilidade de medição e maior facilidade na condução das equipes.

No entanto, assim como as organizações, os processos não são estáticos e necessitam acompanhar a dinâmica das organizações e seus negócios. Daí a importância da melhoria de processos, disciplina que envolve as seguintes etapas: identificação dos pontos fortes, identificação dos problemas que estão interferindo na capacidade do processo, implementação das devidas alterações e avaliação dos impactos e benefícios decorrentes das modificações realizadas.

A norma ISO/IEC 12207 [5] define processo de melhoria como um processo para estabelecer, avaliar, medir, controlar e melhorar um processo de ciclo de vida de software. E a ISO 9000 [6] aponta as seguintes ações como as necessárias para uma melhoria contínua: análise e avaliação da situação existente para identificar áreas para melhorias; estabelecimento 
dos objetivos para melhoria; pesquisa de possíveis soluções para atingir os objetivos; avaliação e seleção destas soluções; implementação da solução escolhida; medição, verificação, análise e avaliação dos resultados da implementação para determinar se os objetivos foram atendidos; e formalização das alterações.

\section{Metodologia de pesquisa}

O objetivo desta pesquisa é identificar as conseqüências do uso de um bom processo de desenvolvimento; as características que devem estar presentes em um bom processo de desenvolvimento; e os aspectos que podem influenciar positiva ou negativamente a utilização do processo.

Como instrumentação para a pesquisa, foi elaborado um questionário que aborda questões a respeito das características e conseqüências de um bom processo de desenvolvimento de software e aspectos que influenciam positiva e negativamente este processo. Tais questões foram implementadas, baseadas na literatura de Engenharia de Software, e não tem a pretensão de ser uma lista completa, podendo o participante da pesquisa inserir novos itens ao questionário. $\mathrm{O}$ questionário foi dividido em cinco partes, sendo que a primeira parte tem como objetivo caracterizar e qualificar o participante em relação à atividade que desempenha, sua formação, sua experiência profissional em projetos de software e seu conhecimento e experiência em Processo de Software. O restante do questionário é formado de questões fechadas com opções múltiplas e exclusivas abrangendo, respectivamente, as conseqüências de um bom processo de desenvolvimento, as características de um bom processo de desenvolvimento e os aspectos que podem influenciar positiva e negativamente a utilização do processo de desenvolvimento de software.

Os questionários foram entregues aos participantes sem que o preenchimento sofresse nenhum tipo de acompanhamento nem controle de tempo de preenchimento. Para tanto, foram utilizados questionários em meio físico, que foram entregues pessoalmente e em meio eletrônico, distribuídos através de e-mail. O preenchimento foi voluntário e realizado no tempo e no ambiente escolhidos pelo participante.

Os indivíduos foram selecionados por conveniência e disponibilidade. No esforço de abranger uma parcela representativa do universo de especialistas em Qualidade e Engenharia de Software no Brasil, o questionário foi distribuído a diversos participantes do II Simpósio Brasileiro de Qualidade de Software (II SBQS); aos participantes do Encontro da Qualidade e Produtividade em Software - EQPS, ambos em Fortaleza, CE; a alunos de pós-graduação e professores da COPPE/UFRJ e a diversas outras pessoas com conhecimento reconhecido na área de Qualidade e Engenharia de Software. Assim sendo, procurou-se obter a colaboração de indivíduos da indústria e da academia, de diversas regiões do país e com experiências diversas com o objetivo de diminuir um possível viés na amostra, decorrente de pontos de vista particulares de cada um desses segmentos.

\section{Consolidação da pesquisa}

A Metodologia e critérios adotados para a consolidação desta pesquisa basearam-se em FARIAS [7] e NOGUEIRA e ROCHA [8] e será vista a seguir.

\subsection{Perfil da amostra inicial}

A primeira parte do questionário tem como objetivo caracterizar e qualificar o participante da pesquisa. De acordo com esta caracterização, será atribuído um peso às suas respostas, uma vez que a amostra utilizada é composta de pessoas de diferentes seguimentos - indústria, 
academia, estudantes - com diferentes graus de conhecimento e experiência. A reunião de todas estas informações permite caracterizar o perfil da amostra inicial. As tabelas de 1 a 6 mostram, para a amostra inicial, respectivamente: Área de Atuação, Atuação em Indústria, Atuação em Universidade, Formação Acadêmica, Conhecimento em Processo de Software e Experiência em Processo de Software. Na tabela 4 foi considerado o nível de formação mais alto, uma vez que o questionário permitia mais de uma escolha.

Tabela 1 - Participantes por Área de Atuação

\begin{tabular}{|l|l|}
\hline Total de Participantes & $\mathbf{9 1}$ \\
\hline Participantes com atuação na indústria & 58 \\
\hline Participantes com atuação na universidade & 67 \\
\hline Participantes com atuação exclusiva na indústria & 24 \\
\hline Participantes com atuação exclusiva na universidade & 33 \\
\hline Participantes com atuação concomitante em ambas & 34 \\
\hline
\end{tabular}

Tabela 2 - Participação por Atuação em Indústria

\begin{tabular}{|l|c|}
\hline Atuação & n \\
\hline Empresário & 5 \\
\hline Gerente de Informática & 2 \\
\hline Gerente da Qualidade & 4 \\
\hline Gerente de Projeto & 11 \\
\hline Analista de Sistemas & 23 \\
\hline Chefe de Desenvolvimento & 2 \\
\hline Consultor TI & 2 \\
\hline Programador & 2 \\
\hline Outros & 12 \\
\hline
\end{tabular}

Tabela 4 - Participantes por Formação Acadêmica

\begin{tabular}{|l|c|c|c|c|}
\hline Nível e Área & Eng. de Software & Computação/Informática & Outro & Total \\
\hline Doutorado & 11 & 4 & 3 & 18 \\
\hline Mestrado & 27 & 10 & 2 & 39 \\
\hline Especialização & 2 & 9 & 8 & 19 \\
\hline Graduação & 0 & 11 & 4 & 15 \\
\hline Total & 40 & 34 & 17 & 91 \\
\hline
\end{tabular}

Tabela 5 - Participantes por Conhecimento em PS Tabela 6 - Participantes por experiência em PS

\begin{tabular}{|l|c|}
\hline Conhecimento & $\mathbf{n}$ \\
\hline Excelente & 9 \\
\hline Alto & 33 \\
\hline Médio & 44 \\
\hline Baixo & 5 \\
\hline Nenhum & 0 \\
\hline
\end{tabular}

\begin{tabular}{|l|c|}
\hline Experiência & n \\
\hline Excelente & 4 \\
\hline Alta & 19 \\
\hline Média & 40 \\
\hline Baixa & 27 \\
\hline Nenhuma & 1 \\
\hline
\end{tabular}

No que se refere ao tempo de atuação na área, o tempo médio foi de 9 anos e a mediana do número de projetos em que participou foi de 7 projetos.

Apesar da distribuição dos questionários ter sido feita em eventos que, por natureza, apresentavam um elevado número de especialistas, não se pode generalizar e acreditar que a 
amostra, na sua totalidade, seja realmente representativa no contexto da pesquisa. Pelo fato dos questionários terem sido distribuídos publicamente em tais eventos, estudantes de graduação e outros profissionais considerados como não-especialistas participaram da pesquisa. Neste sentido, a heterogeneidade da amostra no que tange aos critérios citados acima fica evidenciada nos dados apresentados.

\subsection{Cálculo da Pontuação Individual}

Com o objetivo de diferenciar as respostas dos participantes de acordo com a sua caracterização, a cada indivíduo foi atribuída uma pontuação individual, calculada em função dos diversos fatores que o caracteriza. A formula utilizada para atribuir esta pontuação é:

$P(i)=\operatorname{Max}\{\operatorname{Max}[I(i)] ; \operatorname{Max}[U(i)]\}+F_{1}(i)+F_{2}(i)+T(i)+N(i)+C(i)+E(i)$, onde:

- $\quad P(i)$ é a pontuação total atribuída ao participante $i$;

- $I(i)$ é a pontuação atribuída ao participante $i$ em função de sua atuação na indústria de desenvolvimento de software segundo a tabela 7 ;

- $\quad U(i)$ é a pontuação atribuída ao participante $i$ em função de seu nível de formação acadêmica segundo a tabela 8;

- $\quad F_{1}(i)$ é a pontuação atribuída ao participante $i$ em função de seu nível de formação acadêmico segundo a tabela 9 ;

- $\quad F_{2}(i)$ é a pontuação atribuída ao participante $i$ em função da área de formação acadêmica segundo a tabela 10;

- $\quad T(i)$ é a pontuação atribuída ao participante $i$ em função de seu tempo de atuação na área segunda a tabela 11 ;

- $\quad N(i)$ é a pontuação atribuída ao participante $i$ em função do número de projetos nos quais participou segundo a tabela 12;

- $\quad C(i)$ é a pontuação atribuída ao participante $i$ em função de seu conhecimento em Processo de Software segundo a tabela 13;

- $E(i)$ é a pontuação atribuída ao participante $i$ em função da sua experiência em Processo de Software segunda a tabela 14.

Tabela 7 - Pontuação por atuação na indústria

\begin{tabular}{|l|c|}
\hline Atuação na indústria & I(i) \\
\hline Empresário & 2 \\
\hline Gerente de Informática & 2 \\
\hline Gerente da Qualidade & 4 \\
\hline Gerente de Projeto & 3 \\
\hline Analista de Sistemas & 1 \\
\hline Consultor & 3 \\
\hline
\end{tabular}

Tabela 10 - Pontuação por área de formação

\begin{tabular}{|l|c|}
\hline Área de Formação & $\mathbf{F}_{\mathbf{2}}(\mathbf{i})$ \\
\hline Engenharia de Software & 4 \\
\hline Informática/Computação & 2 \\
\hline Outras áreas & 0 \\
\hline
\end{tabular}

Tabela 8 - Pontuação por atuação na universidade

\begin{tabular}{|l|c|}
\hline Atuação na Universidade & U(i) \\
\hline Professor & 4 \\
\hline Pesquisador & 3 \\
\hline Consultor & 3 \\
\hline Aluno de Doutorado & 2 \\
\hline Aluno de Mestrado & 1 \\
\hline Aluno de Graduação & 0 \\
\hline
\end{tabular}

Tabela 11 - Pontuação por número de projetos

\begin{tabular}{|l|c|}
\hline Número de Projetos & N(i) \\
\hline A partir de 10 & 4 \\
\hline De 4 a 9 & 2 \\
\hline Até 3 & 0 \\
\hline
\end{tabular}

Tabela 9 - Pontuação por nível de formação

\begin{tabular}{|l|c|}
\hline Nível de Formação & F $_{\mathbf{1}}(\mathbf{i})$ \\
\hline Doutorado & 4 \\
\hline Mestrado & 3 \\
\hline Especialização & 2 \\
\hline Graduação & 1 \\
\hline
\end{tabular}

Tabela 12 - Pontuação por tempo de atuação

\begin{tabular}{|l|c|}
\hline Tempo de Atuação & T(i) \\
\hline Mais de 15 anos & 6 \\
\hline Acima de 5, até 15 anos & 3 \\
\hline Até 5 anos & 0 \\
\hline
\end{tabular}


Tabela 13 - Pontuação Conhecimento em Processo de Software

\begin{tabular}{|l|c|}
\hline Conhecimento em Processo de Software & C(i) \\
\hline Excelente & 4 \\
\hline Alto & 3 \\
\hline Médio & 2 \\
\hline Baixo & 1 \\
\hline Nenhum & 0 \\
\hline
\end{tabular}

Tabela 14 - Pontuação Conhecimento em Processo de Software

\begin{tabular}{|l|c|}
\hline Experiência em Processo de Software & E(i) \\
\hline Excelente & 4 \\
\hline Alta & 3 \\
\hline Média & 2 \\
\hline Baixa & 1 \\
\hline Nenhuma & 0 \\
\hline
\end{tabular}

Para que os dados qualitativos fornecidos pelos participantes pudessem ser tratados de forma quantitativa, optou-se pela adoção de tabelas de classes. Para os dados já quantitativos por natureza, a opção de manter as tabelas de classes decorreu do fato de tais itens não serem precisos como, por exemplo: o número de projetos que já participou. A adoção do agrupamento por tabelas de classes permitiu contornar ambos os problemas.

\subsection{Distribuição dos Pesos da Amostra Inicial}

Depois de calculados os pesos dos participantes da amostra inicial, a Distribuição de Freqüência pôde ser determinada, conforme mostrado no gráfico da Figura 1. Dividindo a amostra em quartis, obtêm-se os limites mostrados na Tabela 15.

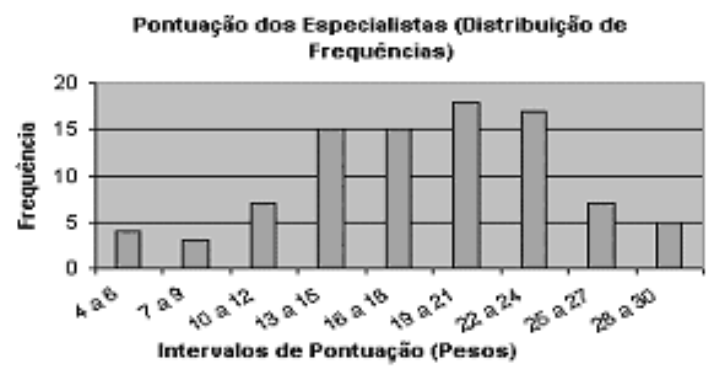

Tabela 15 - Quartis

\begin{tabular}{|l|c|}
\hline Quartis & Pontuação \\
\hline Valor mínimo & 5,0 \\
\hline Limite do $1^{\circ}$ Quartil & 10,0 \\
\hline Limite do $2^{\circ}$ Quartil & 16,0 \\
\hline Limite do $3^{\circ}$ Quartil & 22,0 \\
\hline Limite do $4^{\circ}$ Quartil & 30,0 \\
\hline
\end{tabular}

Figura 1 - Distribuição de Freqüência de Pontuação

\subsection{Ajuste da Amostra Inicial}

Devido ao método de coleta de questionários utilizado, vários dos participantes da amostra inicial não se caracterizam como "especialistas". Os critérios de pontuação adotados permitiram que estes participantes fossem identificados e isolados, sendo representados pelos participantes que compõem o $1^{\circ}$ Quartil da amostra, que foram desconsiderados da amostra no momento da análise de forma a se ter uma melhor representatividade desta.

\subsection{Perfil da Amostra Ajustada}

Uma vez excluídos os participantes que compõem o $1^{\circ}$ Quartil (p(i) $\left.\leq 10,0\right)$, chega-se a um novo perfil para a amostra utilizada na análise. Com o corte, o número de participantes caiu de 91 para 83, porém, a representatividade da amostra foi aumentada.

No que se refere à experiência profissional, o tempo médio de atuação na área aumentou para 9,6 anos e a mediana do número de projetos que o profissional participou aumentou para 8 projetos. 
Tabela 16 - Participantes por Área de Atuação

\begin{tabular}{|l|c|}
\hline Total de Participantes & $\mathbf{8 3}$ \\
\hline Participantes com atuação na indústria & 54 \\
\hline Participantes com atuação na universidade & 60 \\
\hline Participantes com atuação exclusiva na industria & 23 \\
\hline Participantes com atuação exclusiva na universidade & 29 \\
\hline Participantes com atuação concomitante em ambas & 31 \\
\hline
\end{tabular}

Tabela 18 - Participação por Atuação em Universidade

\begin{tabular}{|l|c|}
\hline Atuação & n \\
\hline Professor & 23 \\
\hline Pesquisador & 14 \\
\hline Consultor & 8 \\
\hline Aluno de Doutorado & 11 \\
\hline Aluno de Mestrado & 22 \\
\hline Aluno de Graduação & 1 \\
\hline
\end{tabular}

Tabela 17 - Participação por Atuação em Empresas

\begin{tabular}{|l|c|}
\hline Atuação & n \\
\hline Empresário & 5 \\
\hline Gerente de Informática & 2 \\
\hline Gerente da Qualidade & 4 \\
\hline Gerente de Projeto & 11 \\
\hline Analista de Sistemas & 21 \\
\hline Chefe de Desenvolvimento & 2 \\
\hline Consultor TI & 2 \\
\hline Outros & 11 \\
\hline
\end{tabular}

Tabela 19 - Participantes por Formação Acadêmica

\begin{tabular}{|l|c|c|c|c|}
\hline Atuação/área & Eng. de Software & $\begin{array}{c}\text { Computação } \\
\text { /Informática }\end{array}$ & Outro & Total \\
\hline Doutorado & 11 & 4 & 3 & 18 \\
\hline Mestrado & 27 & 10 & 2 & 39 \\
\hline Especialização & 2 & 9 & 8 & 19 \\
\hline Graduação & 0 & 5 & 2 & 7 \\
\hline Total & 40 & 28 & 15 & 83 \\
\hline
\end{tabular}

Tabela 21 - Participantes por experiência em PS
Tabela 20 - Participantes por Conhecimento em PS

\begin{tabular}{|l|c|}
\hline Conhecimento & n \\
\hline Excelente & 9 \\
\hline Alto & 32 \\
\hline Médio & 41 \\
\hline Baixo & 1 \\
\hline Nenhum & 0 \\
\hline
\end{tabular}

\begin{tabular}{|l|c|}
\hline Experiência & $\mathbf{n}$ \\
\hline Excelente & 4 \\
\hline Alta & 19 \\
\hline Média & 38 \\
\hline Baixa & 22 \\
\hline Nenhuma & 0 \\
\hline
\end{tabular}

As pontuações calculadas para os participantes da amostra ajustada apresentam, agora, a Distribuição de Freqüência apresentada no gráfico da Figura 2.

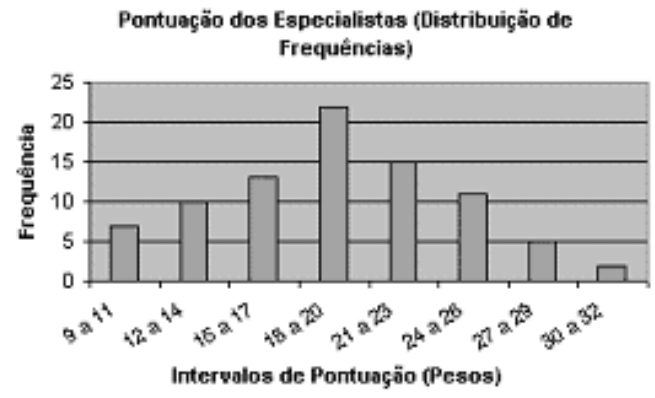

Tabela 22 - Quartis

\begin{tabular}{|l|c|}
\hline Quartis & Pontuação \\
\hline Valor mínimo & 11,0 \\
\hline Limite do $1^{\circ}$ Quartil & 15,0 \\
\hline${\text { Limite do } 2^{\circ} \text { Quartil }}^{\circ}$ & 19,0 \\
\hline${\text { Limite do } 3^{\circ} \text { Quartil }}^{\circ}$ & 24,0 \\
\hline${\text { Limite do } 4^{\circ} \text { Quartil }}^{3}$ & 30,0 \\
\hline
\end{tabular}

Figura 2 -Distribuição de Freqüência de Pontuações

Novamente o agrupamento dos especialistas em classes, adotando-se uma pontuação única para todos aqueles que pertencem à mesma classe foi realizado.

Os Quartis para a amostra ajustada são mostrados na Tabela 22. Na Tabela 23 são apresentadas as classes definidas e seus respectivos valores limite, freqüência (Qtd.), valor médio e desvio padrão. 
Tabela 23 - Classes de especialistas

\begin{tabular}{|c|c|c|c|c|}
\hline Classe & Pontuação Limite & Qtd. & $\begin{array}{c}\text { Valor } \\
\text { Médio }\end{array}$ & $\begin{array}{c}\text { Desvio } \\
\text { Padrão }\end{array}$ \\
\hline A & Até 15,0 & 21 & 13,28 & 1,55 \\
\hline B & De 16,0 a 19,0 & 23 & 17,86 & 1,09 \\
\hline C & De 20,0 a 24,0 & 27 & 22 & 1,46 \\
\hline D & Maior que 24,0 & 12 & 27,16 & 1,69 \\
\hline
\end{tabular}

Tabela 24 - Peso por classe

\begin{tabular}{|c|c|c|}
\hline Classe & Cálculo do Peso & Peso \\
\hline A & 1,0 & 1,00 \\
\hline B & $17,86 / 13,28$ & 1,34 \\
\hline C & $22 / 13,28$ & 1,65 \\
\hline D & $27,16 / 13,28$ & 2,04 \\
\hline
\end{tabular}

Considerando-se a Média e o Desvio Padrão de cada classe, pode-se observar que cada uma apresenta um grau razoável de homogeneidade, o que permite considerar como "não significativa" as diferenças de pesos existentes entre cada um dos componentes.

Para o cálculo dos pesos a serem atribuídos a cada uma das classes, utilizou-se a primeira classe como referência, atribuindo-se a ela um peso igual a 1,0. Para as demais classes, seu peso foi calculado em relação ao peso da primeira classe, de acordo com a formula:

$\mathrm{P}(\mathrm{j})$ é o peso atribuído à classe $\mathrm{j}$;

$$
P(j)=\frac{\bar{X}(j)}{\bar{X}(A)} \quad \text { onde: }
$$

$\mathrm{X}(\mathrm{j})$ é o peso Médio da classe $\mathrm{j}$;

$\mathrm{X}(\mathrm{A})$ é o peso Médio da classe A.

Assim, obtêm-se os pesos de cada classe conforme a tabela 24 .

\subsection{Consolidação dos resultados}

Na consolidação dos resultados, cada voto foi computado de acordo com a classe a que pertence. Para efeito de apresentação dos dados, uma normalização foi aplicada ao total dos votos de cada questão. As Tabelas 25 a 28 apresentam o resultado da consolidação.

$\mathrm{Na}$ Tabela 25 é apresentada a consolidação da pesquisa para as conseqüências de um bom processo de desenvolvimento. As colunas 0,1 e 2 indicam, respectivamente, que o item sendo avaliado nunca está relacionado, às vezes está relacionado ou está muito relacionado.

Tabela 25 - Consolidação dos resultados: Conseqüências de um bom processo de desenvolvimento

\begin{tabular}{|c|c|c|c|}
\hline Item & $\mathbf{0}$ & 1 & 2 \\
\hline Qualidade atingida no produto final & 0,00 & 0,10 & 0,90 \\
\hline Cronograma previsto atingido & 0,00 & 0,42 & 0,58 \\
\hline Custos previstos não ultrapassados & 0,01 & 0,42 & 0,57 \\
\hline Baixo índice de retrabalho & 0,01 & 0,23 & 0,75 \\
\hline Satisfação da equipe & 0,06 & 0,46 & 0,48 \\
\hline Satisfação do cliente & 0,01 & 0,50 & 0,49 \\
\hline Alta produtividade da equipe & 0,02 & 0,42 & 0,56 \\
\hline Produto manutenível & 0,02 & 0,20 & 0,78 \\
\hline Time-to-market & 0,05 & 0,53 & 0,42 \\
\hline Controle gerencial do projeto & 0,00 & 0,15 & 0,85 \\
\hline Relação custo/benefício do uso do processo adequada & 0,01 & 0,28 & 0,71 \\
\hline Riscos gerenciados ao longo do processo de desenvolvimento & 0,01 & 0,25 & 0,74 \\
\hline $\begin{array}{l}\text { Qualidade do processo gerenciada (desvios e não conformidades documentados e } \\
\text { tratados) }\end{array}$ & 0,01 & 0,13 & 0,86 \\
\hline Produtividade da equipe passível de ser medida & 0,00 & 0,33 & 0,67 \\
\hline Satisfação do usuário & 0,05 & 0,55 & 0,40 \\
\hline Melhoria da imagem corporativa da organização & 0,04 & 0,57 & 0,39 \\
\hline Aumento da competitividade da organização & 0,02 & 0,48 & 0,50 \\
\hline Facilidade de adequar-se às mudanças durante o projeto & 0,02 & 0,32 & 0,66 \\
\hline Facilidade de atualização da documentação & 0,03 & 0,30 & 0,67 \\
\hline
\end{tabular}




\begin{tabular}{|l|r|r|r|}
\hline Motivação da equipe de desenvolvimento em utilizar o processo & 0,00 & 0,47 & 0,53 \\
\hline Crescimento no nível de qualificação dos desenvolvedores & 0,03 & 0,37 & 0,60 \\
\hline Promoção de uma cultura comum na organização & 0,00 & 0,20 & 0,80 \\
\hline Menor impacto por rotatividade de pessoal & 0,03 & 0,40 & 0,57 \\
\hline
\end{tabular}

A Tabela 26 apresenta a consolidação da pesquisa para as características de um bom processo de desenvolvimento. As colunas 0,1 e 2 indicam, respectivamente, que o item sendo avaliado não tem importância, tem alguma importância e é muito importante.

Tabela 26 - Consolidação dos resultados: Características de um bom processo de desenvolvimento

\begin{tabular}{|c|c|c|c|}
\hline Item & $\mathbf{0}$ & 1 & 2 \\
\hline Métodos e técnicas adequadas ao projeto & 0,00 & 0,08 & 0,92 \\
\hline Possuir apoio automatizado & 0,04 & 0,51 & 0,45 \\
\hline Fácil de ser utilizado & 0,00 & 0,14 & 0,86 \\
\hline Produzir um volume de documentação adequado ao projeto & 0,00 & 0,36 & 0,64 \\
\hline Processo bem definido & 0,00 & 0,04 & 0,96 \\
\hline Existência de diretrizes para execução das atividades do processo & 0,00 & 0,11 & 0,89 \\
\hline Responsabilidades definidas & 0,00 & 0,07 & 0,93 \\
\hline Entrada/saída de atividades definidas & 0,00 & 0,09 & 0,91 \\
\hline Template de documentos definidos & 0,04 & 0,33 & 0,64 \\
\hline Modelo de ciclo de vida adequado ao projeto & 0,00 & 0,09 & 0,91 \\
\hline Controle da qualidade dos artefatos definido & 0,01 & 0,16 & 0,83 \\
\hline Tipos de testes bem definidos & 0,01 & 0,24 & 0,75 \\
\hline Fornecer conhecimento de experiências em projetos anteriores & 0,00 & 0,43 & 0,57 \\
\hline Adequação ao tamanho e complexidade do projeto & 0,00 & 0,12 & 0,88 \\
\hline Adequação ao tipo de projeto (web, software crítico, etc.) & 0,01 & 0,14 & 0,85 \\
\hline Possuir representação gráfica (visual) do processo & 0,03 & 0,62 & 0,35 \\
\hline Apoio à gerência do projeto & 0,00 & 0,14 & 0,86 \\
\hline Estar baseado nas melhores práticas da Engenharia de Software & 0,01 & 0,23 & 0,76 \\
\hline Aderência à cultura organizacional & 0,01 & 0,25 & 0,73 \\
\hline Permitir avaliação e melhoria de processo & 0,00 & 0,18 & 0,82 \\
\hline Fácil de entender & 0,01 & 0,11 & 0,88 \\
\hline Conformidade com ISO 9000 & 0,10 & 0,63 & 0,26 \\
\hline Conformidade com CMM & 0,05 & 0,61 & 0,34 \\
\hline Granularidade adequada ao desenvolvimento de software & 0,00 & 0,31 & 0,67 \\
\hline Possuir mecanismos para o desenvolvedor fornecer feedback sobre o processo & 0,00 & 0,26 & 0,74 \\
\hline Estar alinhado aos objetivos de negócio da organização & 0,00 & 0,16 & 0,84 \\
\hline Ter sido definido considerando a realidade atual da organização & 0,01 & 0,18 & 0,81 \\
\hline Estar baseado em expectativas realistas & 0,00 & 0,14 & 0,86 \\
\hline Fácil de aprender & 0,02 & 0,26 & 0,73 \\
\hline
\end{tabular}

As Tabelas 27 e 28 apresentam a consolidação da pesquisa para os aspectos que podem influenciar positiva e negativamente a utilização do processo de desenvolvimento. As colunas 0,1 e 2 indicam, respectivamente, que o item sendo avaliado não tem influência, tem alguma influência e tem muita influência.

Tabela 27 - Consolidação dos resultados: Aspectos que podem influenciar positivamente a utilização do processo de desenvolvimento

\begin{tabular}{|c|c|c|c|}
\hline Item & $\mathbf{0}$ & 1 & 2 \\
\hline Equipe experiente & 0,02 & 0,36 & 0,62 \\
\hline Gerência experiente & 0,00 & 0,21 & 0,79 \\
\hline Bom relacionamento com o cliente & 0,09 & 0,51 & 0,40 \\
\hline Existência de apoio automatizado & 0,03 & 0,48 & 0,49 \\
\hline Alto comprometimento da equipe com o projeto & 0,00 & 0,17 & 0,83 \\
\hline Alto comprometimento da gerência com o projeto & 0,00 & 0,12 & 0,88 \\
\hline Treinamento formal da equipe no processo & 0,01 & 0,18 & 0,80 \\
\hline
\end{tabular}




\begin{tabular}{|l|c|c|c|}
\hline Apoio da direção da empresa & 0,00 & 0,09 & 0,91 \\
\hline Motivação da equipe & 0,00 & 0,18 & 0,82 \\
\hline Papéis claramente definidos & 0,00 & 0,12 & 0,88 \\
\hline Estabilidade da equipe & 0,05 & 0,44 & 0,51 \\
\hline $\begin{array}{l}\text { Existência de políticas de incentivo ao uso do processo (ex.: premiações para projetos } \\
\text { com menor número de desvios / não-conformidades) }\end{array}$ & & & \\
\hline Existência de orientações para uso do processo & 0,07 & 0,56 & 0,37 \\
\hline Apoio à utilização do conhecimento de experiências em projetos anteriores & 0,01 & 0,14 & 0,84 \\
\hline Ambiente físico de trabalho adequado & 0,00 & 0,38 & 0,62 \\
\hline Boa infra-estrutura de apoio (equipamentos, software, rede, biblioteca, etc.) & 0,01 & 0,47 & 0,52 \\
\hline Existência de um Grupo de Processo de Engenharia de Software na empresa (SEPG) & 0,00 & 0,31 & 0,69 \\
\hline Sensibilização na empresa da importância do uso do processo & 0,04 & 0,45 & 0,51 \\
\hline Aderência do processo à cultura organizacional & 0,00 & 0,12 & 0,88 \\
\hline Existência de auditorias da aderência ao processo & 0,00 & 0,19 & 0,81 \\
\hline Estimativas realistas para o projeto & 0,02 & 0,43 & 0,55 \\
\hline Clareza do que pode ser melhorado na organização & 0,01 & 0,16 & 0,83 \\
\hline Seguir rigorosamente uma abordagem de processo (ISO, CMM, etc.) & 0,04 & 0,35 & 0,62 \\
\hline Ter claro que se quer atingir um nível mais alto de maturidade ou certificação ISO & 0,12 & 0,56 & 0,32 \\
\hline Iniciar a implantação do processo pelas atividades mais fáceis & 0,07 & 0,60 & 0,33 \\
\hline Iniciar a implantação do processo pelas atividades mais relevantes & 0,14 & 0,62 & 0,24 \\
\hline Disciplina na implantação das atividades & 0,06 & 0,34 & 0,61 \\
\hline O processo estar alinhado aos objetivos de negócio da organização & 0,02 & 0,13 & 0,85 \\
\hline O processo estar baseado em expectativas realistas & 0,00 & 0,13 & 0,87 \\
\hline $\begin{array}{l}\text { O processo considerar a complexidade e o tempo necessário para mudanças culturais } \\
\text { efetivas }\end{array}$ & 0,00 & 0,10 & 0,90 \\
\hline
\end{tabular}

Tabela 28 - Consolidação dos resultados: Aspectos que podem influenciar negativamente a utilização do processo de desenvolvimento

\begin{tabular}{|c|c|c|c|}
\hline Item & $\mathbf{0}$ & 1 & 2 \\
\hline Mudanças nos objetivos da organização & 0,00 & 0,44 & 0,56 \\
\hline Falta de apoio da direção da organização & 0,00 & 0,12 & 0,88 \\
\hline Perda de atualidade do processo & 0,03 & 0,39 & 0,57 \\
\hline Inexistência de políticas de incentivo ao uso do processo & 0,04 & 0,50 & 0,46 \\
\hline Equipe do projeto mal dimensionada & 0,04 & 0,33 & 0,63 \\
\hline Equipe com perfil inadequado ao projeto & 0,02 & 0,18 & 0,80 \\
\hline Inadequação às características dos desenvolvedores & 0,01 & 0,30 & 0,69 \\
\hline Falta de comunicação entre os membros da equipe & 0,02 & 0,19 & 0,80 \\
\hline Falta de um bom relacionamento entre os desenvolvedores e o gerente & 0,02 & 0,24 & 0,74 \\
\hline Falta de um envolvimento continuado dos interessados & 0,00 & 0,22 & 0,78 \\
\hline Grande número de mudanças de requisitos no projeto & 0,07 & 0,36 & 0,56 \\
\hline Resistências à implantação do processo & 0,00 & 0,17 & 0,83 \\
\hline Não institucionalização do processo & 0,00 & 0,27 & 0,73 \\
\hline Alta rotatividade da direção da organização & 0,09 & 0,39 & 0,52 \\
\hline Estimativa de custo/prazo não realistas & 0,01 & 0,24 & 0,75 \\
\hline Pouca experiência da equipe & 0,04 & 0,57 & 0,39 \\
\hline Pouca experiência da gerência & 0,02 & 0,40 & 0,58 \\
\hline Problemas no relacionamento da equipe & 0,02 & 0,37 & 0,61 \\
\hline Falta de apoio automatizado & 0,07 & 0,62 & 0,31 \\
\hline Falta de comprometimento da equipe & 0,00 & 0,11 & 0,89 \\
\hline Falta de comprometimento da gerência & 0,00 & 0,02 & 0,98 \\
\hline Falta de treinamento no processo & 0,00 & 0,19 & 0,81 \\
\hline Falta de coordenação e liderança nas atividades de implantação & 0,00 & 0,12 & 0,88 \\
\hline Inadequação às características dos projetos & 0,00 & 0,26 & 0,74 \\
\hline Desconsiderar a complexidade e o tempo necessário para mudanças culturais efetivas & 0,00 & 0,23 & 0,77 \\
\hline Não ter claro que se quer atingir um nível mais alto de maturidade ou certificação ISO & 0,20 & 0,61 & 0,20 \\
\hline Alta rotatividade de desenvolvedores & 0,08 & 0,42 & 0,51 \\
\hline
\end{tabular}


\begin{tabular}{|l|r|r|r|}
\hline Alta rotatividade de gerentes & 0,02 & 0,31 & 0,67 \\
\hline
\end{tabular}

\section{Análise dos resultados}

Os resultados apresentados na seção anterior foram muito interessantes, por tornar claras as concepções que a indústria e academia têm em relação a processos de desenvolvimento de software. Como veremos a seguir, algumas estão de acordo com o que foi evidenciado em estudos encontrados na literatura. Em outras, percebem-se contradições.

No primeiro subgrupo de itens: "Conseqüências de um bom processo de desenvolvimento", pôde-se identificar o que a comunidade acredita serem os principais resultados que um bom processo de desenvolvimento é capaz de atingir. O resultado mostra que se acredita que a maior conseqüência é a "Qualidade atingida no produto final", estando de acordo com a evidência da forte relação entre a qualidade do produto final e a qualidade do processo utilizado. Segundo FLORAC e CARLETON [9], para melhorar a qualidade deve-se focar nos processos que geram os produtos e serviços.

A próxima conseqüência mais bem avaliada foi a "Qualidade do processo gerenciada (desvios e não conformidades documentados e tratados)". Esta avaliação mostra a compreensão de que quanto melhor um processo, melhor a sua gerência de qualidade. Em terceiro lugar apresentou-se a consequência: "Controle gerencial do projeto". Isto denota a concepção de que um bom processo de desenvolvimento de software auxilia sobremaneira na gerência dos vários fatores envolvidos em um projeto de software.

PFLEEGER [10] apóia as avaliações anteriores ao definir que quanto maior a maturidade do processo, maior a sua visibilidade, fornecendo um conjunto mais rico de informações e um melhor entendimento do mesmo.

De acordo com os participantes da pesquisa, o menor resultado obtido com a implantação de um bom processo de desenvolvimento de software é a "Melhoria da imagem corporativa da organização". E a segunda conseqüência foi a "Satisfação do usuário".

Estes dois resultados demonstram uma não compreensão da interdependência dos vários efeitos que um bom processo de desenvolvimento de software pode gerar, pois os mesmos participantes elegeram a "Qualidade atingida no produto final" como sendo a maior consequência de um bom processo. Porém se uma organização melhora o seu produto final, melhorará também a satisfação dos seus usuários, melhorando a imagem corporativa da organização no mercado.

"Time-to-market", ou seja, a rapidez em colocar um novo produto ou versões no mercado foi a terceira conseqüência menos considerada. A avaliação deste item está em desacordo com alguns trabalhos da literatura que mostram a importância de um bom processo para o aumento da produtividade e diminuição do retrabalho, que estão intimamente relacionados à velocidade de entrega de produtos ao mercado.

Algo que pode ter acontecido é o item ter sido mal compreendido pelos participantes da pesquisa. Além disto, podem ter raciocinado que uma empresa, apesar de não ter um bom processo, pode conseguir colocar seus produtos em tempo hábil no mercado, mesmo em detrimento da qualidade do produto.

Outro subgrupo apresenta o que a comunidade considera serem características de um bom processo de desenvolvimento, ou seja, os critérios e seus valores que podem classificá-lo como um bom processo. A principal característica considerada foi "Processo bem definido", evidenciando que os participantes da pesquisa acreditam que para um processo de desenvolvimento ser bom de se utilizar, deve ser definido criteriosamente. Segundo GRUHN [11], uma boa modelagem de um processo de software é fundamental para facilitar a sua implantação e utilização. 
A segunda característica melhor avaliada foi "Responsabilidades definidas". Há um entendimento da importância das responsabilidades estarem claramente definidas. EMAM [12] destacou que um dos elementos mais importantes para a infra-estrutura de um processo é ter as responsabilidades bem definidas, confirmando a avaliação realizada.

A característica "Métodos e técnicas adequadas ao projeto" foi a terceira melhor avaliada. Esta característica diz respeito à existência de diferenças entre projetos e da necessidade de tratá-los de forma diferente. OLIVEIRA [13] define como um dos passos para a definição de um processo de software, identificar os métodos e ferramentas utilizadas na organização para o tipo de software a ser desenvolvido.

LEUNG e YUEN [14] realçaram esta importância ao apresentarem um relato de experiência de uma empresa em Hong Kong que começou por definir um processo padrão para todos os projetos da organização, apesar de $80 \%$ dos seus projetos serem pequenos. Observou-se depois a necessidade de adaptar o processo padrão para pequenos projetos, pois estava havendo um aumento das atividades administrativas e de documentação.

As próximas duas características melhor avaliadas foram: "Modelo de ciclo de vida adequado ao projeto" e "Entrada/saída de atividades definidas". Estas dizem respeito à importância da escolha de um modelo de ciclo de vida adequado ao projeto, que é uma das decisões mais difíceis de serem tomadas, e à relevância do processo de desenvolvimento definir claramente os artefatos de entrada e saída para cada atividade.

As características com avaliação mais baixa, em ordem decrescente, foram: "Conformidade com ISO 9000", "Conformidade com CMM" e "Possuir representação gráfica (visual) do processo". Este resultado mostra que a comunidade não considera ser relevante para a qualidade de um processo de desenvolvimento estar em conformidade com as normas ISO 9000 e CMM, normas estas desenvolvidas por organizações de renome e cuja certificação ou atestado está sendo buscado, cada vez mais, pelas empresas em âmbito internacional.

Para HEFNER e TAUSER [15] é estrategicamente importante definir claramente os objetivos da organização, não tendo como principal objetivo apenas conseguir mais um nível de maturidade. MESSNARZ [16] destacou que as orientações dadas pelas metodologias para avaliações, tipo CMM, nem sempre estão de acordo com os objetivos da empresa.

Considerou-se, também, que ter uma representação gráfica de um processo não significa necessariamente que este é bom.

O terceiro subgrupo a ser analisado contém os aspectos que podem influenciar positivamente a utilização de um processo de desenvolvimento. Com isto buscou-se conhecer os aspectos que estão muito relacionados ao sucesso da utilização de um processo.

O aspecto considerado como mais importante foi o "Apoio da direção da empresa". Acredita-se, portanto, que o envolvimento da alta direção é extremamente relevante para a utilização do processo de software em uma organização, o que confirma a literatura.

$\mathrm{O}$ segundo aspecto identificado como de muita relevância foi "O processo estar baseado em expectativas realistas". Constatou-se que a comunidade acredita que um processo deve ser definido especificamente para uma organização, baseando-se em expectativas que estejam dentro do real e do possível. FLORAC e CARLETON [9] compactuaram deste argumento ao dizerem que quando da definição, implementação e manutenção de um processo de software, deve-se garantir que a empresa tem habilidade para executar e manter os processos.

Os aspectos: "Alto comprometimento da gerência com o projeto", "Papéis claramente definidos" e "Sensibilização na empresa da importância do uso do processo", ficaram empatados como terceiro aspecto mais importante. Evidencia-se a importância que é dada ao comprometimento do gerente de um projeto com o processo de desenvolvimento de software, bem como a compreensão, por parte da equipe do projeto, da relevância para um projeto de 
software em seguir um processo de desenvolvimento. $O$ outro aspecto mostrou ser considerado importante para a utilização do processo: ter cada um dos participantes conhecimento claro de seus papéis.

KALTIO e KINNULA [17] destacaram a relevância de sensibilizar a empresa a respeito da importância de se utilizar processos de software, ao apresentarem um estudo de caso ocorrido na empresa Nokia Mobile Phones Ltd. onde o último elemento do framework de implantação do processo de desenvolvimento foi a atividade de promoção do processo, que foi fundamental para o sucesso do projeto de implantação.

Os três aspectos considerados como os que têm menos influência positiva na utilização do processo foram: "Iniciar a implantação do processo pelas atividades mais fáceis", "Seguir rigorosamente uma abordagem de processo (ISO, CMM, etc.)" e "Ter claro que se quer atingir um nível mais alto de maturidade ou certificação ISO”. A comunidade considerou como não sendo relevante iniciar pelas atividades mais fáceis e nem seguir rigorosamente uma abordagem de avaliação de processo. Além disto, não se viu muita relação entre a facilidade de utilizar um processo de desenvolvimento com a clareza em atingir um nível mais alto de maturidade ou certificação ISO. CATTANEO et al. [18] confirmaram a pequena influência do item "Seguir rigorosamente uma abordagem de processo (ISO, CMM, etc.)" na utilização de um processo, ao descreverem a experiência de uma empresa em que não foi suficiente usar apenas o CMM, sendo importante analisar, detalhadamente, os problemas organizacionais e estratégicos da empresa.

O último subgrupo diz respeito aos aspectos que podem afetar negativamente a utilização do processo de desenvolvimento, ou seja, aqueles fatores que devem ser bem gerenciados para que a equipe utilize bem e de forma adequada o processo implantado.

O aspecto considerado mais crítico foi o "Falta de comprometimento da gerência", mostrando que os participantes da pesquisa consideram que o fato da gerência não estar comprometida com o processo como sendo o maior risco para o sucesso da sua utilização.

O segundo aspecto considerado crítico foi "Falta de comprometimento da equipe". Este resultado mostra a importância do comprometimento com o processo, daqueles que fazem parte do projeto. HEFNER e TAUSER [15] confirmaram a relevância do comprometimento da equipe ao destacarem o cuidado que a gerência deve ter na fase de execução do processo, para não dificultar o envolvimento dos participantes e para não negligenciar a necessidade de treinamentos.

Os aspectos "Falta de apoio da direção da organização" e "Falta de coordenação e liderança nas atividades de implantação" também foram tidos como influenciando bastante negativamente a utilização do processo. Estes dizem respeito à importância da direção da organização apoiar a utilização de processos e de a atividade de implantação do processo em um projeto, ser bem coordenada e liderada.

Os três aspectos considerados como de menor risco para o sucesso na utilização de um processo de software foram: "Não ter claro que se quer atingir um nível mais alto de maturidade ou certificação", "Falta de apoio automatizado" e "Pouca experiência da equipe". Estes resultados significam que os participantes consideram que, não ter claro que se quer atingir um nível maior de maturidade ou certificação ISO e contar com uma equipe pouco experiente tem pouco efeito negativo na utilização do processo. Além disto, a falta de apoio automatizado ao processo também não é um fator de muita influência negativa para a sua utilização.

A visão de EMAM [12] em relação ao item "Pouca experiência da equipe" é contrária ao resultado da pesquisa, já que o autor ressalta a necessidade de se dispor de pessoal competente para a infra-estrutura de um processo. FLORAC e CARLETON [9] também têm a mesma 
opinião ao definirem como sendo, pessoal mal treinado, uma das causas que podem interferir na variação de um processo.

\section{Conclusões e trabalhos futuros}

Este trabalho apresentou o resultado de uma pesquisa, caracterizando concepções que a indústria e a academia, no contexto brasileiro, têm em relação a processos de desenvolvimento de software. Com isto, tornou-se possível compreender as possíveis motivações que estão por trás de um projeto de implantação e melhoria de processos de software, bem como as características de qualidade de um processo e os aspectos que podem influenciar positiva ou negativamente na utilização deste processo.

Observou-se que, para implantar e utilizar um processo de software é necessário considerar vários fatores, conhecer o peso de cada um deles e o tipo de relações existentes entre eles. Com isto, pode-se obter um direcionamento melhor que ajude a ter mais sucesso na sua utilização, auxiliar a administração dos principais riscos envolvidos na utilização do processo, bem como conhecer os possíveis resultados de uma melhoria. Além disto, pode ajudar a priorizar as melhorias a serem implantadas, baseando-se nos pesos de cada fator.

Como trabalhos futuros, espera-se realizar outra pesquisa que possibilite identificar as relações entre os diversos itens que foram identificados, quantificando a força da relação, bem como o grau de incerteza, de modo a gerar um diagrama de influências que auxilie na construção de modelos de simulação a serem implementados em uma ferramenta. Com isto, buscar-se-á apoiar, de forma mais simples, os gerentes de projetos a anteciparem possíveis tendências durante o processo e a priorizar as melhorias a serem implantadas no processo de desenvolvimento.

\section{Agradecimentos}

Os autores agradecem ao CNPq e a CAPES pelo apoio financeiro ao projeto. Agradecem também a todos os participantes da pesquisa e a Mauro Oddo Nogueira.

\section{Referências}

1. REED, K., 2000, Software engineering - a new millenium?, IEEE Software, JulyAugust.

2. MCT/SEPIN - Secretaria de Política de Informática e Automação / Ministério da Ciência e Tecnologia, 2002, Qualidade e Produtividade no Setor de Software Brasileiro - 2001 / n.4., Brasília.

3. PAULK, M. C., CURTISS, B., CHRISSIS, M. B., WEBER, C. V., 1993, Capability Maturity Model for Software, Version 1.1, Pittsburgh, Software Engineering Institute.

4. ZAHRAN, S., 1997, Software Process Improvement - Practical Guidelines for Business Success; Addison-Wesley.

5. ISO/IEC 12207, 1995, ISO/IEC 12207. Information technology - software life cycle, International Standard Organization.

6. ISO 9000, 2000, Quality Management Systems - Fundamental and Vocabulary.

7. FARIAS, L. L., 2002, Planejamento de Riscos em Ambientes de Desenvolvimento de Software Orientados à Organização, Tese de M.SC., COPPE/UFRJ, Rio de Janeiro, Brasil. 
8. NOGUEIRA, M. O., ROCHA, A. R., 2003, Práticas Relevantes em Engenharia de Software: uma avaliação de especialistas, II Simpósio Brasileiro de Qualidade de Software, Fortaleza, CE, Brasil.

9. FLORAC, W., CARLETON, A. E., 2000, Measuring the Software Process: Statistical Process Control for Software Process Improvement, Addison-Wesley.

10. PFLEEGER, S. L., 2000, Improving Predictions, Products, Processes and Resources, In: Software Engineering, cap.13, pp. 563-592.

11. GRUHN, V., 2000, Software Process Landscaping, Software Process: Improvement an Practice, 5:111-120.

12. EMAM, K. E., 2001, Software Engineering Process, in IEEE - Trial Version.

13. OLIVEIRA, K. M., 1999, Modelo para Construção de Ambientes de Desenvolvimento de Software Orientados a Domínio, Tese de D.SC., COPPE/UFRJ, Rio de Janeiro, RJ, Brasil.

14. LEUNG, H. K. N., YUEN, T. C. F., 2001, A Process Framework for Small Projects, Software Process: Improvement an Practice, 6:67-83.

15. HEFNER, R., TAUSER, J., 2001, Things They Never Taught You in CMM School, 26th Annual NASA Goddard Software Engineering Workshop, November, pp. 27-29.

16. MESSNARZ, R., 1999, Road map for Readers and How to Use the Book, In: Richard Messnarz and Colin Tully (eds.), Better Software Practice for Business Benefit: Principles and Experience, IEEE Computer Society, cap1, pp.1-13.

17. KAlTIO, T., KINNUlA, A., 2000, Deployng the Defined SW Process, Software Process: Improvement and Practice, 5:65-83.

18. CATTANEO, F., FUGGETA, A., SCIUTO, D., 2001, Pursuing Coherence in Software Process Assessment and Improvement, Software Process: Improvement and Practice, $6: 3-22$. 